\title{
Ferric carboxymaltose versus ferric gluconate in hemodialysis patients: reduction of erythropoietin dose in 4 years of follow-up
}

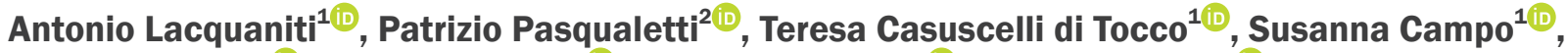 \\ Stefania Rovito ${ }^{1(\mathbb{D})}$, Maurizio Bucca ${ }^{1(\mathbb{D})}$ Antonino Ragusa $^{1(\mathbb{D})}$, Paolo Monardo ${ }^{1(\mathbb{D})}$ \\ 'Nephrology and Dialysis Unit, Papardo Hospital, Messina, Italy \\ ${ }^{2}$ Service of Medical Statistics \& IT (SeSMIT), Fatebenefratelli Foundation for Health Research and Education, Rome, Italy
}

Background: Ferric carboxymaltose (FCM) is a parenteral, dextran-free iron formulation designed to overcome the limitations of existing iron preparations. The main aim of this study was to retrospectively examine results obtained from a long period of FCM therapy in hemodialysis patients who have been previously treated with ferric gluconate (FX). Markers of iron metabolism, erythropoietin (EPO) doses, and effects on anemic status have been analysed.

Methods: The study was performed with a follow up period of 4 years, when patients were treated before with FX and then switched to FCM. A total of 25 patients were included in the study.

Results: FCM increased transferrin saturation (TSAT) levels by $11.9 \%(P<0.001)$ with respect to FX. Events of TSAT less than $20 \%$ were reduced during FCM. The monthly dose of EPO was reduced in the FCM period $(-6,404.1$ international unit [IU]; 95\% confidence interval, $-10,643.5 \mathrm{IU} ;-2,164.6 \mathrm{IU} ; P=0.003$ ), as well as the erythropoietin resistance index $(P=0.004)$. During the period with $\mathrm{FCM}$, ferritin levels were higher than during $\mathrm{FX}(P<0.001)$, while transferrin was reduced $(P=0.001)$.

Conclusion: During FCM treatment, minor doses of EPO were administered if compared to those delivered during FX therapy. Stable and on target levels of hemoglobin were maintained with better control of anemia through high levels of ferritin and TSAT.

Keywords: Anemia, Erythropoietin resistance index, Ferric carboxymaltose, Hemodialysis

Received January 24, 2020; Revised May 7, 2020;

Accepted May 10, 2020

Editor: Seung-Yeup Han, Keimyung University, Daegu, Republic of Korea

Correspondence: Paolo Monardo

Nephrology and Dialysis Section, Papardo Hospital, Messina 98100, Italy. E-mail: pmonardo66@gmail.com

Copyright (C) 2020 by The Korean Society of Nephrology

(a) This is an open-access article distributed under the terms of the Creative Commons Attribution Non-Commercial License (http://creativecommons. org/licenses/by-nc-nd/4.0/), which permits unrestricted non-commercial use, distribution, and reproduction in any medium, provided the original work is properly cited.

\section{Introduction}

Iron deficiency is common in the non-dialysis nephropathic population, and it is even more pronounced in patients on hemodialysis (HD). Intravenous (IV) iron therapy is recommended in these patients, due to the frequent blood loss associated with dialysis machine use and inability to achieve optimum outcomes with oral iron [1]. Moreover, in HD patients, a functional iron deficiency coexists, characterized by unsuccessful mobilization of iron from stores. This is associated with normal or high ferritin levels and low transferrin saturation (TSAT) values, indicating limited transport of iron to the erythron for erythropoiesis. The inflammatory state is asso- 
ciated with increased serum hepcidin, which blocks iron absorption from the duodenum and iron release from the liver (main iron store) and macrophages, thereby interrupting iron recycling [2]. The decreased availability of iron for erythropoiesis aggravates the existing anemia of chronic disease (functional iron deficiency) in HD patients.

Currently, available IV iron agents vary in indication, dosing regimens, and safety profiles, and there are concerns about related anaphylactic reactions due to dextran-containing iron formulations. Current IV iron formulations include high or low molecular weight iron dextran, ferric gluconate (FX), iron sucrose, and ferric carboxymaltose (FCM). In particular, FCM (Ferinject ${ }^{\circledR}$; Vifor (International) Inc., St Gallen, Switzerland) is a next-generation parenteral, dextran-free iron formulation designed to overcome the limitations of existing IV iron preparations.

IV iron agents share the same core chemistry, but they differ in the size of the core and the identity and density of the surrounding carbohydrate. These differences determine pharmacologic and biologic effects, such as clearance rate after injection, iron release rate, bioactiv- ity, and maximum tolerated dose. Whereas FX is structurally based on a weak complex of carbohydrate shell, which is rapidly degraded in blood, FCM is characterized by a more stable structure. FCM is partially broken after administration in the blood, inducing a gradual release of iron and avoiding precocious TSAT (Fig. 1).

Studies on pharmacodynamics and pharmacokinetics with radio-labelled FCM demonstrated iron uptake by the reticulo-endothelial system (RES) and delivery to the endogeneous transport system for heme synthesis in new erythrocytes with minimal release of free iron [3]. Considering the RES as a peripheral compartment, a 2-compartment model was the best fit for FCM, which may be taken up by hepatocytes or return to circulation as intact complexes [4,5]. No information is available on the pharmacokinetics of FX at dosage of $>125 \mathrm{mg}$ [6]. Although FCM is an attractive option in terms of both efficacy and safety, widespread use of this formulation is not yet supported by a high level of evidence. Clinical trials of FCM versus comparators conducted in over 10,000 patients with iron deficiency anemia and associated with a variety of medical conditions have shown improvements in hemoglobin $(\mathrm{Hb})$ levels and replenishment of depleted iron

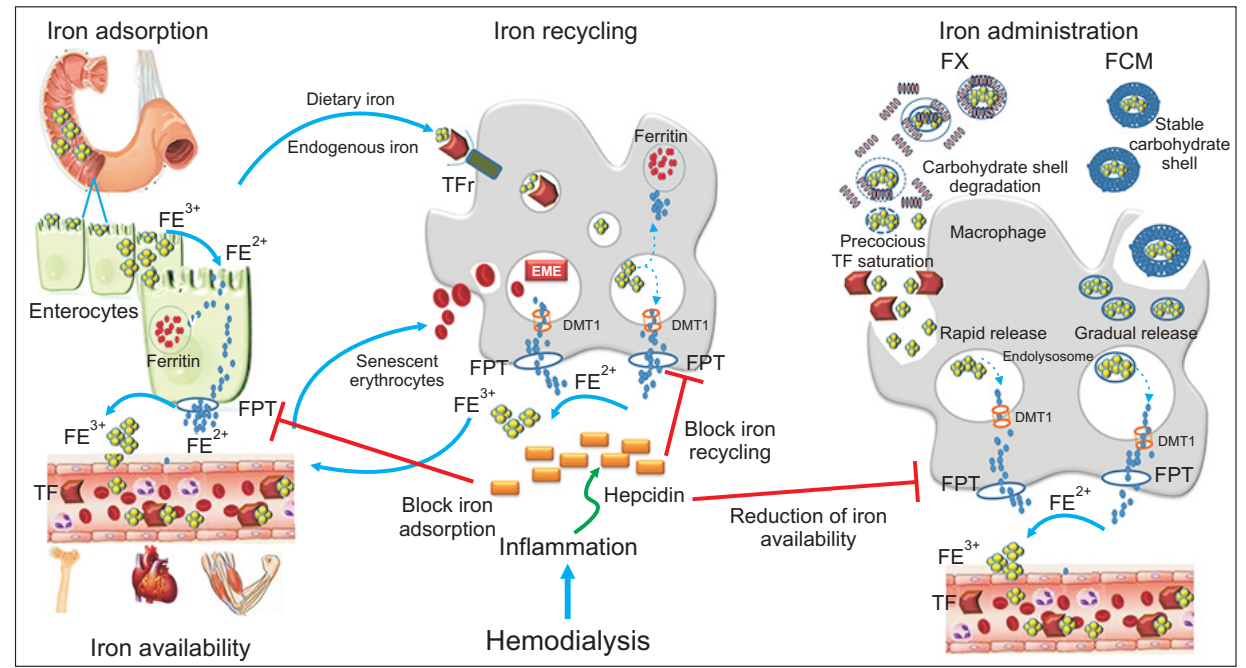

Figure 1. Endogenous iron metabolism and iron administration. Dietary $\mathrm{Fe}^{3+}$ is first reduced to $\mathrm{Fe}^{2+}$, which then enters the intestinal cell where it is either stored as ferritin or it leaves the cell via ferroportin (FPT). Macrophages catch iron from endogenous sources, such as dietary iron via enterocytes and senescent erythrocytes, release $\mathrm{Fe}^{3+}$ through FPT, and play a pivotal role in iron recycling. Ferric gluconate (FX) is structurally characterized by a weak complex of carbohydrate shell that is rapidly degraded in blood, whereas ferric carboxymaltose (FCM) is characterized by a more stable carbohydrate shell that is only partially broken after administration in blood. The carbohydrate shell is completely broken inside macrophages, and the iron core is included in endolysosomes. This process results in gradual and more stable release of iron into the blood, avoiding precocious transferrin saturation (TSAT). Hepcidin, a peptide hormone synthesized by liver cells, negatively regulates all these processes, blocking FPT. This figure is original and entirely realized by the authors.

DMT 1, divalent metal transporter 1; TF, transferrin; TFr, TF receptor. 
stores $[7,8]$.

Several studies have been also conducted in the nephropathic population, but the cohort was often affected by chronic kidney disease not on dialysis. The dialytic population was enrolled to analyze the safety and effects of the drug, but there are no data about its superiority versus other iron IV formulations [9-11].

The most appropriate IV iron-replacement regimen in adults undergoing dialysis is unknown, which has resulted in different local, national, and international recommendations and practices. Kidney Diseases Outcomes Quality Initiative (KDOQI) recommended IV iron to maintain $\mathrm{Hb}$ between 11 and $12 \mathrm{~g} / \mathrm{dL}$, ferritin between 100 and $800 \mathrm{ng} / \mathrm{mL}$, and TSAT between $20 \%$ and 50\% [12].

Observational studies have raised concern that monthly doses of $300 \mathrm{mg}$ or more of IV iron are associated with poor outcomes. Recently, a multicenter, open-label trial showed that among HD patients, the use of proactively administered high-dose IV iron resulted in a significantly lower dose of erythropoietin (EPO) and a lower incidence of blood transfusion than the use of a low-dose regimen administered reactively. Mortality and incidence of nonfatal cardiovascular events and infections did not differ significantly between the two treatment groups [13].

The main aim of this study was to retrospectively examine results obtained from a long period of FCM therapy in HD patients who were previously treated with FX. Markers of iron metabolism, variation of EPO administration, and effects on anemic status were analyzed. FCM was hypothesized to be superior to FX.

\section{Methods}

\section{Study design}

A retrospective study was performed with a follow up period of 4 years. The enrollment phase started in January 2014 with patients treated with IV ferric gluconate (Ferlixit, FX; Aventis Pharma, Milan, Italy) in the past for $\geq 6$ months. This phase lasted for 24 months until December 2015, when FX was discontinued. In this period, $125 \mathrm{mg}$ of gluconate iron was administered per week. During January 2016, a wash out period was performed. From February 2016 to February 2018, patients were treated with FCM.

Patients received an undiluted dose of FCM directly into the venous line of the dialyzer $\sim 30$ to 60 minutes into the dialysis session. Hb, TSAT, and ferritin levels were evaluated in accordance with the KDOQI guidelines [14]. IV iron therapy was initiated when there was an absolute iron deficiency associated with the EPO dose, defined by TSAT $<20 \%$ or ferritin $<200 \mathrm{ng} / \mathrm{mL}$. During FX treatment, all patients required $125 \mathrm{mg}$ per week to maintain adequate iron metabolism. Conversely, patients received $100 \mathrm{mg}$ of FCM per week after the switch.

Fig. 2 demonstrates the applied algorithm for mainte-

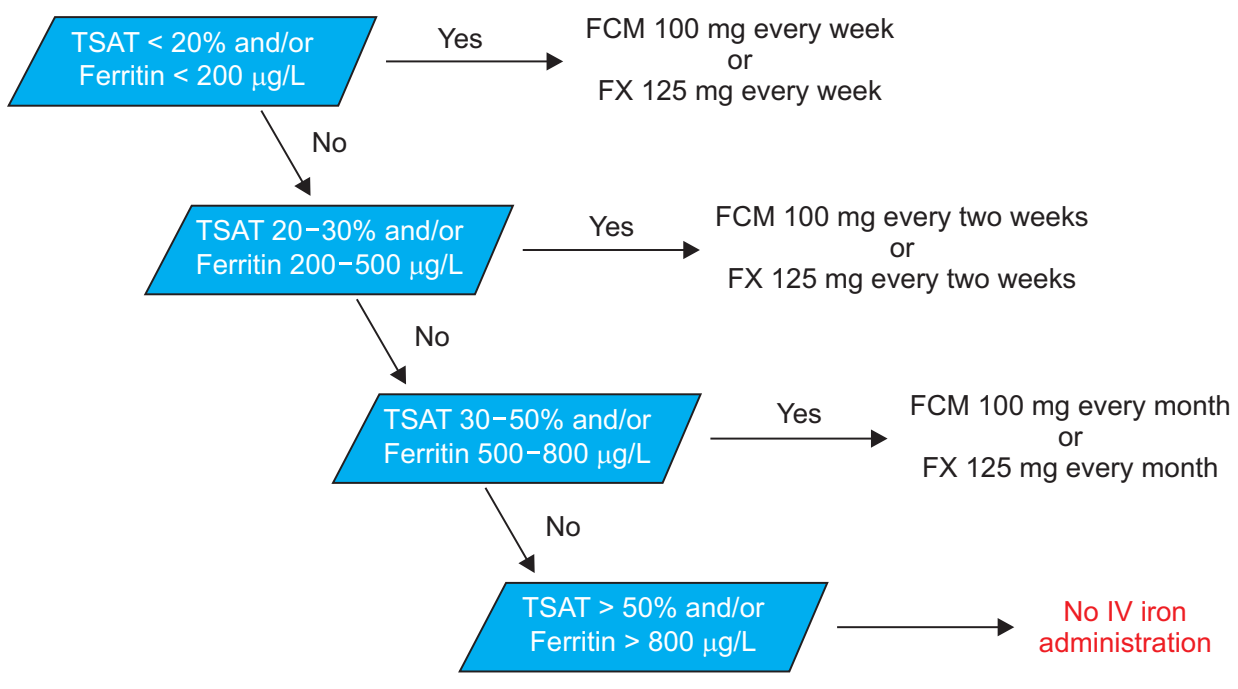

Target values
Figure 2. Flow diagram of patient selection.

FCM, ferric carboxymaltose; FX, ferric gluconate; Hb, hemoglobin; IV, intravenous; TSAT, transferrin saturation. 
nance therapy according to $\mathrm{Hb}$, TSAT, and ferritin levels.

\section{Patients and baseline data}

A total of 64 patients who were receiving maintenance HD as outpatients at the Papardo Hospital, Nephrology and Dialysis Unit, Messina, Italy, were screened.

All patients received HD three times a week for 4 hours each. Patients were at least 18 years old, were on uninterrupted HD treatment for a period of at least 6 months before the switch from FX to FCM, and continued the treatment for at least 12 months.

Patients with any malignancy diagnosed in the five years prior to the investigational period were excluded. Moreover, other exclusion criteria were hospitalization during the 6 months prior to enrollment, hospitalization during the study period, history of gastro-intestinal bleeding, history of hemotransfusion, $\mathrm{Hb}$ concentration more than $12.0 \mathrm{~g} / \mathrm{dL}$, known hypersensitivity to any component of FCM, anemia other than that due to iron and EPO deficiency, evidence of active infection (including hepatitis B, hepatitis C, or human immunodeficiency virus), concomitant severe liver or cardiovascular diseases, and iron storage disorders.

According to the abovementioned criteria, 25 patients were included in the study (Fig. 3).

The study was approved by the Ethics Committee for Medical Research of the University Hospital of Messina, Italy and carried out in accordance with the Declaration of Helsinki. All patients provided informed consent permitting data sampling and analysis at the time of initiat- ing dialysis therapy.

\section{Blood collection and biochemical data}

Data were measured once per month, and blood sampling was performed at the beginning of each week when initiating HD.

TSAT was calculated according to the following formula: (serum iron/serum transferrin) $\times 70.9$.

The erythropoietin resistance index (ERI) was defined as the weekly weight-adjusted EPO dose (U/ kg/wk) divided by the $\mathrm{Hb}$ level (g/dL). ERI was calculated monthly to investigate resistance to EPO treatment [15].

\section{Statistical methods}

\section{Justification of sample size}

According to previously available data, the EPO dose administered to HD patients was about $30,000 \mathrm{U}$ with variability (standard deviation) of 11,000 U. In a pilot sample, the correlation between mean EPO doses in two different periods 6 months apart was quite high $(r=0.81)$. For caution, we assumed a slightly lower correlation $(\mathrm{r}=$ 0.75), thus making the a priori assumption that about $50 \%$ (49\%) of variance in EPO dose at T1 can be explained by the EPO dose used 6 months before T0. Considering the paired design and the within-subjects planned analysis, we computed that a sample of 21 patients allows reaching a power of $80 \%$ to detect a statistically significant (with two-tailed alpha level set at 0.05) difference between treatments of 5,000 U. This difference was con-
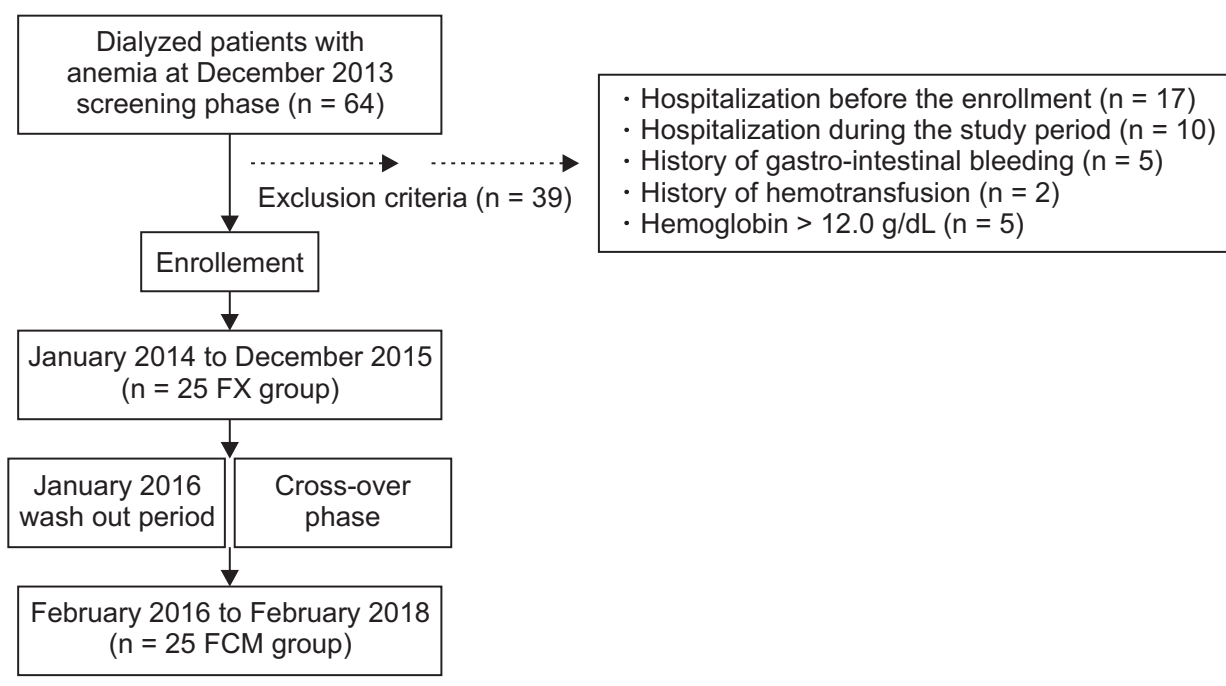

Figure 3. Study design: inclusion and exclusion criteria, enrollment, and cross over phase. FCM, ferric carboxymaltose; FX, ferric gluconate. 
sidered the "minimal relevant difference" in the sense that lower values are deemed unimportant in terms of pharmaeconomics.

\section{Statistical analysis}

The retrospective study was designed as a paired twoperiod study in which each patient was individually controlled, but without the possibility of a further control group.

According to the "long" structure of the dataset (several rows for each patient corresponding to monthly visits), the first analysis was a General Estimating Equation (GEE) model with "Patient" as a random-subjects variable, "Time" and "Treatment" as repeated variables within patients, and all measures of interest as dependent variables. Moreover, simpler statistical analyses were also performed by applying paired $t$ tests and the Wilcoxon signed-rank nonparametric test. For this purpose, median and interquartile range was computed for every parameter and for each patient and treatment period.

Multiple regression analyses were performed by constructing a model including all univariate correlates of $\mathrm{Hb}$ levels to assess independent relationships. Data were expressed as partial correlation coefficients $(\beta)$ and $P$ values.

\section{Results}

\section{Patients baseline characteristics}

The main baseline characteristics of the study cohort are summarized in Table 1. The mean age of patients was $62.4 \pm 19.6$ years. The median value for dialysis vintage was 77 months (range 52-179). The primary disease was diabetic nephropathy in 10 patients $(40.0 \%)$, nephrosclerosis in 8 patients (32.0\%), chronic glomerulonephritis in 4 patients $(16.0 \%)$, and other causes in the 3 remaining patients $(12.0 \%)$.

Dialysis sessions lost 4 hours with a mean weekly spKt/ $\mathrm{V}$ of $1.46 \pm 0.3$. The mean $\mathrm{Hb}, \mathrm{Fe}$, ferritin, and TSAT were $11.73 \pm 1.4 \mathrm{~g} / \mathrm{dL}, 91.9 \pm 43.2 \mu \mathrm{g} / \mathrm{dL}, 522.5(378-750) \mathrm{ng} /$ $\mathrm{mL}$, and $21.3 \pm 8.9$, respectively. The mean monthly EPO dose was $29,666.66 \pm 20,731.54$ IU.
Table 1. Baseline characteristics of study cohort at enrollment

\begin{tabular}{|c|c|}
\hline Characteristic & Value $(n=25)$ \\
\hline Age (yr) & $62.4 \pm 19.6$ \\
\hline Male/female & $19(76.0) / 6(24.0)$ \\
\hline \multicolumn{2}{|l|}{ Comorbidities } \\
\hline Diabetes mellitus & $12(48.0)$ \\
\hline Hypertension & $21(84.0)$ \\
\hline Current smokers & $5(20.0)$ \\
\hline Heart failure & $6(24.0)$ \\
\hline \multicolumn{2}{|l|}{ Primary renal disease } \\
\hline Diabetic nephropathy & $10(40.0)$ \\
\hline Nephrosclerosis & $8(32.0)$ \\
\hline Chronic glomerulonephritis & $4(16.0)$ \\
\hline Others (nephrolithiasis, infectious disease) & $3(12.0)$ \\
\hline \multicolumn{2}{|l|}{ Laboratoristic parameters } \\
\hline Albumin (g/dL) & $3.45 \pm 0.10$ \\
\hline Hemoglobin (g/dL) & $11.73 \pm 1.40$ \\
\hline Parathyroid hormone $(\mathrm{pg} / \mathrm{mL})$ & $375.0 \pm 121.6$ \\
\hline Ferritin $(\mathrm{ng} / \mathrm{mL})$ & $522.5(378.0-750.0)$ \\
\hline Transferrin (mg/dL) & $121.5 \pm 87.5$ \\
\hline TSAT (\%) & $21.3 \pm 8.9$ \\
\hline EPO dose (monthly IU) & $29,666.66 \pm 20,731.54$ \\
\hline ERI (EPO dose/Hb) & $2,711.26 \pm 2,091.10$ \\
\hline \multicolumn{2}{|l|}{ Dialytic parameters } \\
\hline Time per session $(\mathrm{h})$ & $4.13 \pm 0.27$ \\
\hline $\mathrm{Qb}(\mathrm{mL} / \mathrm{min})$ & $280.8 \pm 63.5$ \\
\hline Arteriovenous fistula & $20(80)$ \\
\hline $\mathrm{spKt} / \mathrm{V}$ & $1.46 \pm 0.30$ \\
\hline Dialysis vintage $(\mathrm{mo})^{a}$ & $77(52-179)$ \\
\hline
\end{tabular}

Data are presented as mean \pm standard deviation, number (\%), or median (interquartile range).

EPO, erythropoietin; ERI, erythropoietin resistance index; Hb, hemoglobin; IU, international unit; Qb, blood flow rate; TSAT, transferrin saturation.

${ }^{\mathrm{a}}$ Median (range).

\section{Comparison between FCM and FX treatment}

The main findings are reported in Table 2. No side effects were recorded during the entire study period. The monthly dose of EPO was clearly reduced in the FCM period $(-6,404.1 ; 95 \%$ confidence interval $[\mathrm{CI}],-10,643.5$ to $-2,164.6 ; P=0.003)$ compared to EPO doses delivered to the FX group. Moreover, patients receiving FCM were characterized by higher levels of iron $(+8.0$; 95\% CI, -1.3 to $+17.2 ; P=0.087)$ and ferritin $(+162.4 ; 95 \% \mathrm{CI},+79.5$ to $+245.2 ; P<0.001)$ than during $\mathrm{FX}$, while transferrin values were reduced $(-19.8 ; 95 \% \mathrm{CI},-32.2$ to $-7.4 ; P=$ 0.001). Furthermore, FCM increased TSAT levels of $11.9 \%$ 
Lacquaniti, et al. Ferric carboxymaltose in hemodialysis

Table 2. Comparison between FCM and FX treatment

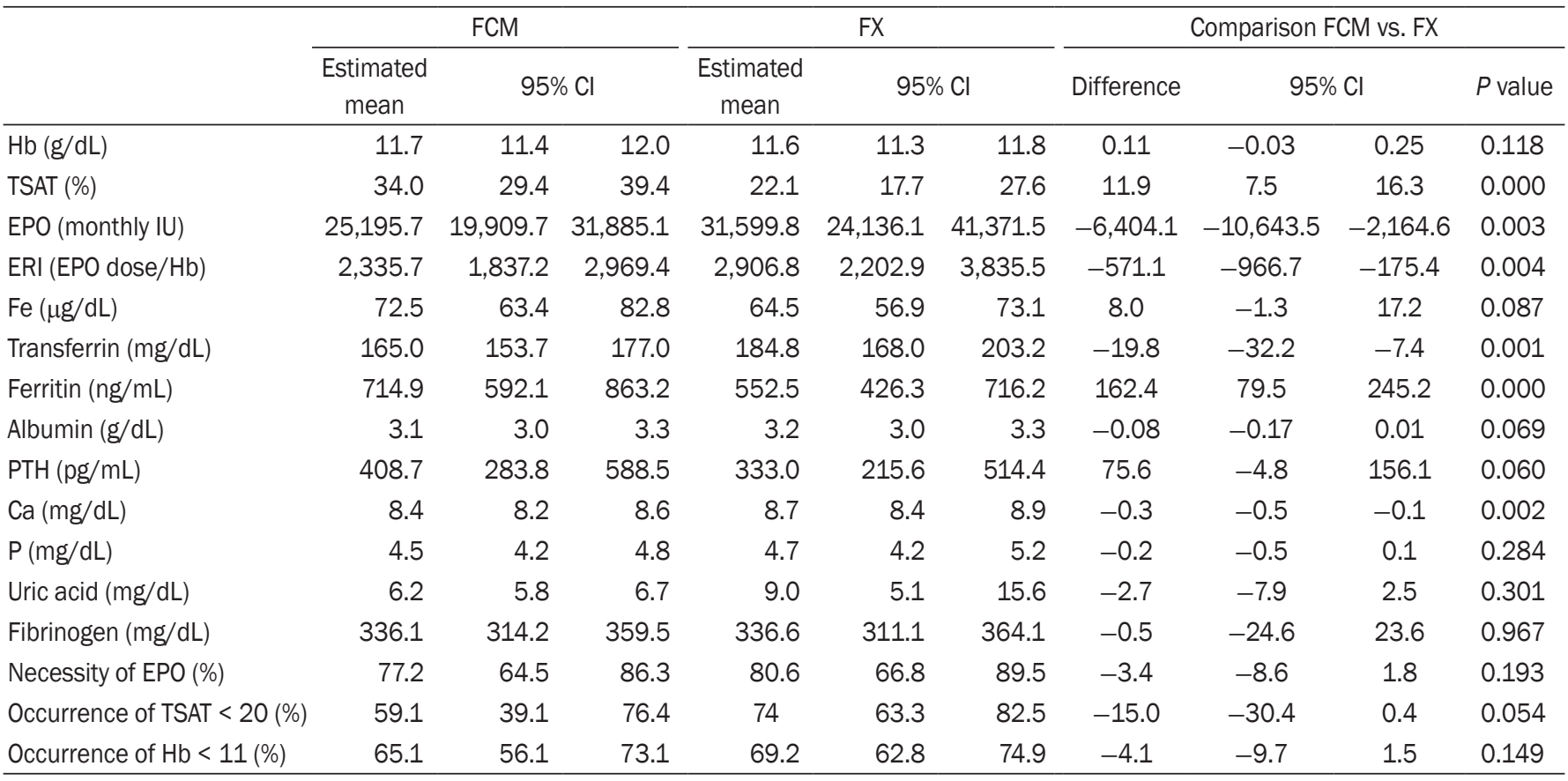

Ca, serum calcium; Cl, confidence interval; FCM, ferric carboxymaltose; Fe, iron; FX, iron gluconate; EPO, erythropoietin; ERI, erythropoietin resistance index; Hb, hemoglobin; IU, international unit; P, serum phosphorus; PTH, parathyroid hormone; TSAT, transferrin saturation.

$(95 \% \mathrm{CI},+7.5$ to $+16.3 ; P<0.001)$ with respect to $\mathrm{FX}$.

No differences were recorded between the two treatment periods for $\mathrm{Hb}(P=0.118)$, although FCM therapy allowed maintenance of $\mathrm{Hb}$ values with fewer units of EPO. Incidence of TSAT less than $20 \%$, indicating severe iron deficiency, was also reduced during FCM therapy $(-15.0 \% ;-30.4 \%$ to $+0.4 \% ; P=0.054)$. The FCM group was characterized by significant reduction of the ratio of EPO dose/Hb (ERI), (-571.1; 95\% CI, -966.7 to $-175.4 ; P=$ 0.004) compared with the FX group (Fig. 4).

Finally, we assessed whether the most relevant differences between treatments in terms of iron balance (TSAT) and monthly EPO dose remained significant after considering inflammatory status. After entering $\mathrm{Ca}$, parathyroid hormone (PTH), P, and fibrinogen as covariates, the difference in TSAT between FCM and FX remained significant $(+11.1 ; 95 \% \mathrm{CI},+3.2$ to $+19.1 ; P=0.005)$. Similarly, the adjustment for inflammatory indexes did not substantially change the treatment difference in monthly EPO dose $(-10,726.9 ; 95 \% \mathrm{CI},-20,496.2$ to $-957.6 ; P=$ 0.028).

Since these findings were obtained using an advanced procedure capable of managing clusters (in our case, a cluster is a patient) and within-subjects correlated data, their robustness was assessed by applying basic statistical procedures to aggregated data. More specifically, medians were computed for each combination patient/period and then a paired $t$ test was applied to each parameter. Very similar findings were obtained, confirming the general superiority of FCM to FX treatment. These results are reported in Table 3.

The nonparametric Wilcoxon signed-rank test was applied as a further check, and all results were confirmed.

\section{Univariate correlations of $\mathrm{Hb}$ levels and multiple regression analysis}

In univariate analysis, $\mathrm{Hb}$ levels, recorded during FCM treatment, were directly correlated with albumin $(\mathrm{r}=$ $0.13 ; P=0.0001)$, serum PTH $(\mathrm{r}=0.13 ; P=0.001)$, calcium phosphorus product ( $\mathrm{r}=0.6 ; P=0.002)$, and transferrin $(\mathrm{r}=$ $0.10 ; P=0.02)$, whereas a significant inverse correlation was found with serum iron ( $\mathrm{r}=-0.12 ; P=0.0001)$, ferritin $(\mathrm{r}=-0.12 ; P=0.002)$, EPO monthly dose $(\mathrm{r}=-0.71 ; P<$ $0.0001)$, and ERI ( $\mathrm{r}=-0.78 ; P=0.0001$ ) (Fig. 5).

In contrast, no significant correlation was found between $\mathrm{Hb}$ levels and other clinical and laboratory parameters, such as uric acid and TSAT ( $\mathrm{r}$ range $=0.03$ to $0.10, P>$ 

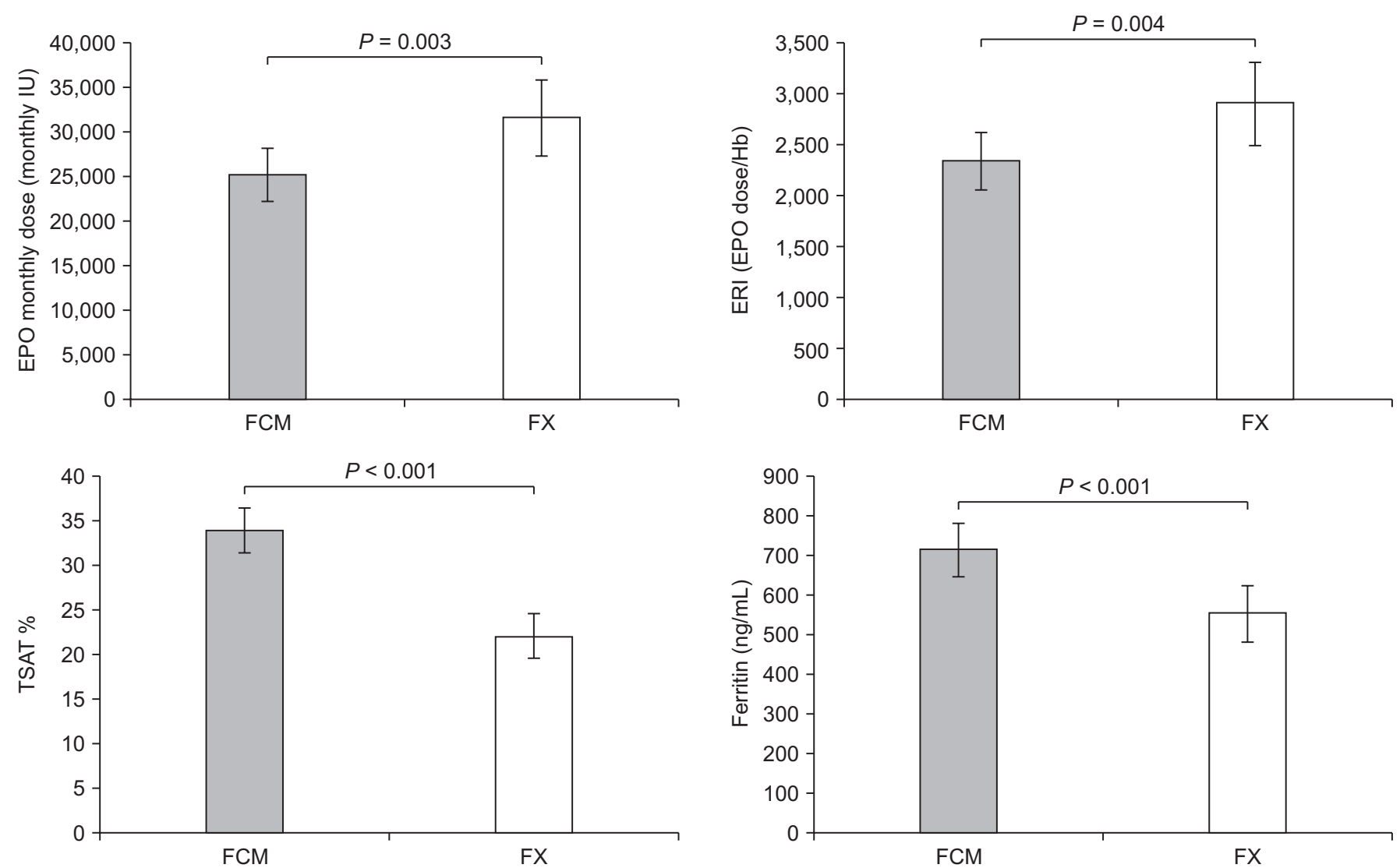

Figure 4. Differences between ferric carboxymaltose and ferric gluconate.

FCM, ferric carboxymaltose; FX, ferric gluconate; IU, international unit.

Table 3. Comparison between FCM and FX treatment: Supplemental statistical analysis

\begin{tabular}{|c|c|c|c|c|c|c|}
\hline \multirow[b]{3}{*}{$\mathrm{Hb}(\mathrm{g} / \mathrm{dL})$} & \multicolumn{6}{|c|}{ FCM FX Comparison FCM vs. FX } \\
\hline & \multirow{2}{*}{$\begin{array}{c}\text { Mean } \pm \text { SD } \\
11.7 \pm 0.8\end{array}$} & \multirow{2}{*}{$\begin{array}{l}\text { Mean } \pm \text { SD } \\
11.6 \pm 0.8\end{array}$} & \multirow{2}{*}{$\begin{array}{c}\text { Difference } \\
0.15\end{array}$} & \multicolumn{2}{|c|}{$95 \% \mathrm{Cl}$ of the difference } & \multirow{2}{*}{$\begin{array}{c}P \text { value } \\
0.139\end{array}$} \\
\hline & & & & -0.05 & 0.35 & \\
\hline EPO (monthly IU) & $25,069.1 \pm 15,202$ & $32,852.4 \pm 22,473.7$ & $-7,783.3$ & $-12,866.2$ & $-2,700.4$ & 0.004 \\
\hline ERI (EPO dose/Hb) & $2,201 \pm 1,374.3$ & $2,936.4 \pm 2,084.8$ & -735.4 & $-1,198.8$ & -271.9 & 0.003 \\
\hline $\mathrm{Fe}(\mu \mathrm{g} / \mathrm{dL})$ & $68.7 \pm 24.4$ & $56.2 \pm 17.6$ & 12.6 & 2.9 & 22.3 & 0.013 \\
\hline Ferritin $(\mathrm{ng} / \mathrm{mL})$ & $772.5 \pm 377.7$ & $474.4 \pm 361.8$ & 298.1 & 150 & 446.2 & 0.000 \\
\hline Albumin (g/dL) & $3.1 \pm 0.3$ & $3.2 \pm 0.4$ & -0.1 & -0.2 & 0 & 0.018 \\
\hline PTH (pg/mL) & $365.7 \pm 406.5$ & $283 \pm 293.1$ & 82.7 & -7.8 & 173.3 & 0.072 \\
\hline $\mathrm{Ca}(\mathrm{mg} / \mathrm{dL})$ & $8.4 \pm 0.5$ & $8.7 \pm 0.6$ & -0.3 & -0.6 & -0.1 & 0.003 \\
\hline $\mathrm{P}(\mathrm{mg} / \mathrm{dL})$ & $4.6 \pm 1.2$ & $4.7 \pm 1.3$ & -0.1 & -0.5 & 0.3 & 0.667 \\
\hline Occurrence TSAT < 20 (\%) & $23.9 \pm 25.9$ & $47.9 \pm 47.7$ & -24.1 & -39.7 & -8.4 & 0.004 \\
\hline Occurrence HB < $11(\%)$ & $27.7 \pm 15.8$ & $32.4 \pm 22.4$ & -4.7 & -12.2 & 2.8 & 0.211 \\
\hline
\end{tabular}

Ca, serum calcium; FCM, ferric carboxymaltose; Fe, iron; FX, iron gluconate; EPO, erythropoietin; ERI, erythropoietin resistance index; Hb, hemoglobin; IU, international unit; P, serum phosphorus; PTH, parathyroid hormone; SD, standard deviation; TSAT, transferrin saturation. 

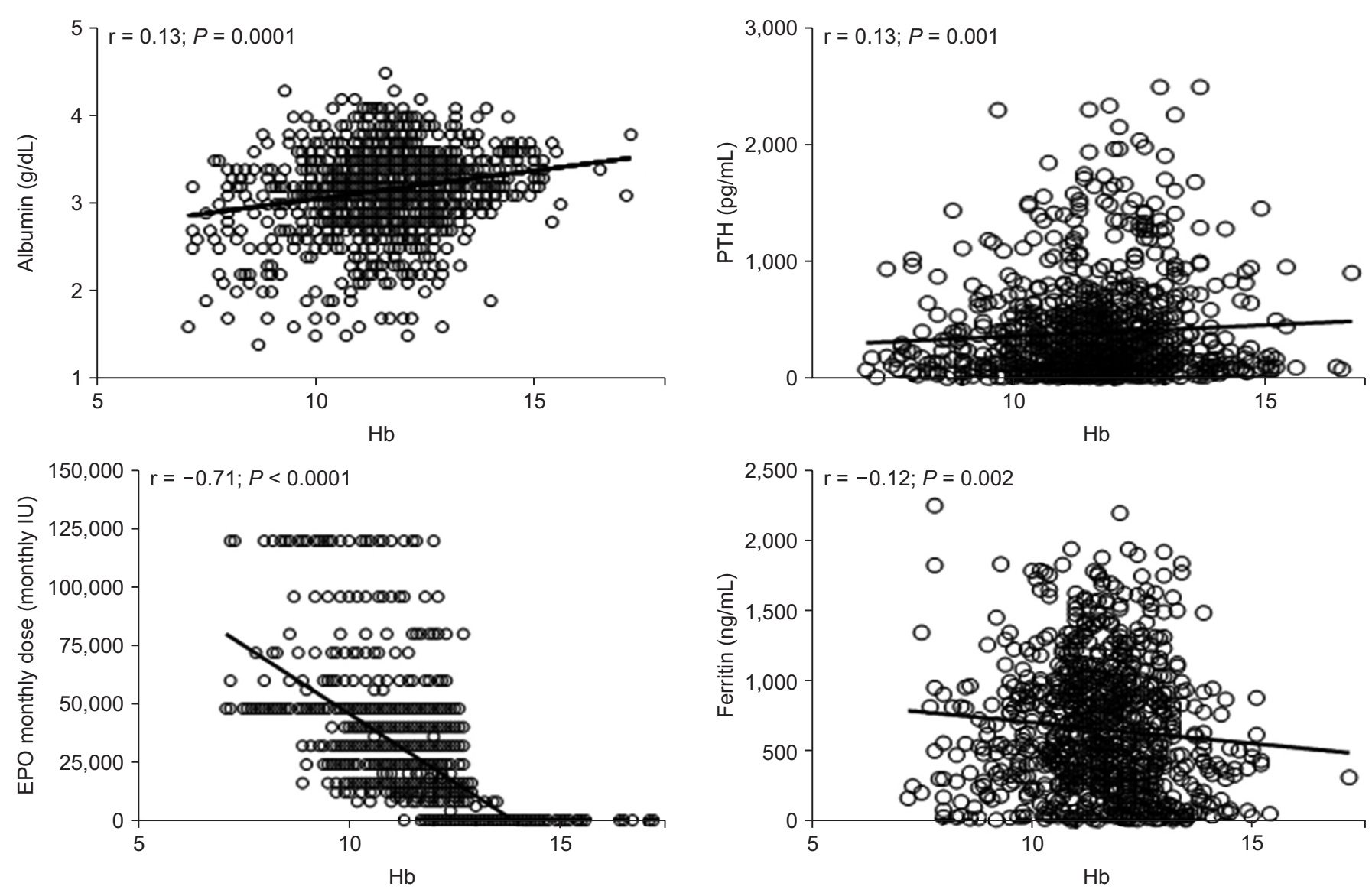

Figure 5. Univariate relationships of hemoglobin $(\mathrm{Hb})$ levels in hemodialyzed patients.

IU, international unit.

0.06).

All variables found to be significantly related to $\mathrm{Hb}$ values in univariate analysis were introduced in a multivariate model using $\mathrm{Hb}$ level as a dependent variable. After adjustment for other factors, significance was maintained for the correlation between $\mathrm{Hb}$ and albumin, monthly dose of EPO, ERI, and ferritin. In contrast, the correlations with calcium-phosphorus product and PTH found in univariate analysis were lost.

\section{Discussion}

Our study revealed that treatment with FCM achieved target levels of $\mathrm{Hb}$ with reduced dosage of EPO. The FCM cohort was characterized by a reduced percentage of patients with TSAT $<20 \%$, compared to the FX group, revealing better control of iron homeostasis and increased serum levels of iron and ferritin.

Hofman et al [16] confirmed our data and demon- strated the superiority of FCM compared to iron sucrose in obtaining better iron status in HD patients. Moreover, this was the first study comparing FCM and FX.

The increased ferritin and TSAT values observed during FCM therapy are related to improved bioavailability of elemental iron and a gradual, stable release of iron into the blood, avoiding precocious TSAT $[5,17]$.

In the FCM group, our data revealed a reduced number of patients with $\mathrm{Hb}$ levels $<11 \mathrm{~g} / \mathrm{dL}$, defined as the target level. This is clinically relevant because the proportion of patients who achieve this value in a single month is usually $30 \%$ or less [1].

This important clinical target was obtained not only through improved iron management, but also by administering fewer monthly doses of EPO in the FCM group, which has positive economic implications. Reduced EPO doses may also be related to the results of several randomized controlled trials which showed that better patient outcomes are not exclusively and closely related to 
high levels of $\mathrm{Hb}$ induced by EPO administration, which may also be harmful [18-20].

Whereas nutritional status did not change during the entire observational period and no differences in albumin levels were detected after the therapy switch, reduced ERI levels were detected in the FCM cohort, demonstrating the response to EPO.

ERI represents an indirect index of inflammation. Ferritin and TSAT, the currently recommended tests to assess iron status in HD patients, have limitations that make diagnosis of iron deficiency or overload challenging [1]. Moreover, alterations in calcium and phosphorous metabolism linked to secondary hyperparathyroidism and vascular calcifications are closely related to chronic inflammation [21]. In our study, we did not evaluate modifications of inflammatory biomarkers, such as $\mathrm{C}$ reactive protein. However, the reduced levels of ERI in the FCM cohort may represent a link between FCM and inflammation. Further studies are necessary to confirm our hypothesis about the relationship between FCM and effects on ERI.

Compared to other IV iron preparations, FCM did not lead to additional complement activation during HD, revealing its low pro-inflammatory activities [22].

Several reports have demonstrated a strong relationship between FCM and phosphate metabolism that is mediated by complex interactions between iron formulations and fibroblast growth factor (FGF)-23, which determines hypophosphataemia [23]. FGF-23 acts primarily on the kidney to down-regulate expression of sodiumphosphate co-transporters and stimulate phosphaturia. A randomized controlled trial showed that FCM resulted in a transient increase in FGF23 through inhibition of its degradation and a subsequent increased rate of urinary phosphate excretion [24]. Our data did not assess modifications in phosphate levels between the two groups because iron-induced hypophosphatemia was not observed in our dialyzed population, in which kidney function was completely lost. The phosphaturic effects of FGF-23 were also not studied because all patients had anuria.

Finally, this study demonstrates that FCM, administered by bolus-push injection into the HD venous line, is a well-tolerated therapy with no adverse reaction recorded during the entire studied period.

The present study has some limitations. First, it was a single-center, retrospective study, and the patient cohort was relatively small with confounding factors that may have affected $\mathrm{Hb}$ levels. Although the target $\mathrm{Hb}$ level was set, anemia therapy depended on the discretion of each doctor. Given these limitations, a prospective study on the effects of FCM on Hb levels in HD patients is required in the future. Confirmation in wider cohorts is indispensable to verify the general validity to our report. Second, C-reactive protein, an inflammatory marker used to ascertain functional iron deficiency, was not included. However, we did account for a number of speculative inflammatory markers, including serum albumin, ERI and ferritin, obtaining interesting data for this study generating hypotheses.

In conclusion, we have clearly demonstrated that minor doses of EPO administered during FCM treatment, compared to those delivered during FX therapy, help maintain stable and target levels of Hb. Better control of anemia was also achieved, as demonstrated by high levels of ferritin and TSAT.

Further observational and prospective studies are warranted to comprehensively determine the factors that influence anemia and iron homeostasis and their implications on inflammation and outcomes in HD patients.

\section{Conflicts of interest}

All authors have no conflicts of interest to declare.

\section{Authors' contributions}

Antonio Lacquaniti, Patrizio Pasqualetti, and Paolo Monardo designed the study, and analysed and interpreted the data. Teresa Casuscelli Di Tocco, Stefania Rovito, and Susanna Campo drafted the article. Maurizio Bucca and Antonino Ragusa revised the paper. All authors provided intellectual content of critical importance to the work. All authors finally approved this version of the manuscript for publication.

\section{References}

[1] Kidney Disease: Improving Global Outcomes (KDIGO) Anemia Work Group. KDIGO clinical practice guideline for anemia in chronic kidney disease. Kidney Int Suppl 2012;2: 279-335.

[2] Eleftheriadis T, Liakopoulos V, Antoniadi G, Kartsios C, Ste- 
fanidis I. The role of hepcidin in iron homeostasis and anemia in hemodialysis patients. Semin Dial 2009;22:70-77.

[3] Lyseng-Williamson KA, Keating GM. Ferric carboxymaltose: a review of its use in iron-deficiency anaemia. Drugs 2009;69:739-756.

[4] Muñoz M, Olsen PS, Petersen TS, Manhart S, Waldorff S. Pharmacokinetics of ferric bepectate-a new intravenous iron drug for treating iron deficiency. Basic Clin Pharmacol Toxicol 2019;125:133-141.

[5] Danielson BG. Structure, chemistry, and pharmacokinetics of intravenous iron agents. J Am Soc Nephrol 2004;15 Suppl 2:S93-S98.

[6] Seligman PA, Dahl NV, Strobos J, et al. Single-dose pharmacokinetics of sodium ferric gluconate complex in irondeficient subjects. Pharmacotherapy 2004;24:574-583.

[7] Charytan C, Bernardo MV, Koch TA, Butcher A, Morris D, Bregman DB. Intravenous ferric carboxymaltose versus standard medical care in the treatment of iron deficiency anemia in patients with chronic kidney disease: a randomized, active-controlled, multi-center study. Nephrol Dial Transplant 2013;28:953-964.

[8] Covic A, Mircescu G. The safety and efficacy of intravenous ferric carboxymaltose in anaemic patients undergoing haemodialysis: a multi-centre, open-label, clinical study. Nephrol Dial Transplant 2010;25:2722-2730.

[9] Onken JE, Bregman DB, Harrington RA, et al. Ferric carboxymaltose in patients with iron-deficiency anemia and impaired renal function: the REPAIR-IDA trial. Nephrol Dial Transplant 2014;29:833-842.

[10] Qunibi WY, Martinez C, Smith M, Benjamin J, Mangione A, Roger SD. A randomized controlled trial comparing intravenous ferric carboxymaltose with oral iron for treatment of iron deficiency anaemia of non-dialysis-dependent chronic kidney disease patients. Nephrol Dial Transplant 2011;26:1599-1607.

[11] Macdougall IC, Bock AH, Carrera F, et al. FIND-CKD: a randomized trial of intravenous ferric carboxymaltose versus oral iron in patients with chronic kidney disease and iron deficiency anaemia. Nephrol Dial Transplant 2014;29: 2075-2084

[12] National Kidney Foundation. IV. NKF-K/DOQI clinical practice guidelines for anemia of chronic kidney disease: update 2000. Am J Kidney Dis 2001;37(1 Suppl 1):S182-
S238.

[13] Macdougall IC, White C, Anker SD, et al.; PIVOTAL Investigators and Committees. Intravenous iron in patients undergoing maintenance hemodialysis. N Engl J Med 2019;380: 447-458.

[14] KDOQI. KDOQI clinical practice guideline and clinical practice recommendations for anemia in chronic kidney disease: 2007 update of hemoglobin target. Am J Kidney Dis 2007;50:471-530.

[15] Locatelli F, Andrulli S, Memoli B, et al. Nutritional-inflammation status and resistance to erythropoietin therapy in haemodialysis patients. Nephrol Dial Transplant 2006;21: 991-998.

[16] Hofman JMG, Eisenga MF, Diepenbroek A, et al. Switching iron sucrose to ferric carboxymaltose associates to better control of iron status in hemodialysis patients. BMC Nephrol 2018;19:242.

[17] Geisser P, Burckhardt S. The pharmacokinetics and pharmacodynamics of iron preparations. Pharmaceutics 2011;3: 12-33.

[18] Pfeffer MA, Burdmann EA, Chen CY, et al.; TREAT Investigators. A trial of darbepoetin alfa in type 2 diabetes and chronic kidney disease. N Engl J Med 2009;361:2019-2032.

[19] Singh AK, Szczech L, Tang KL, et al.; CHOIR Investigators. Correction of anemia with epoetin alfa in chronic kidney disease. N Engl J Med 2006;355:2085-2098.

[20] Drüeke TB, Locatelli F, Clyne N, et al.; CREATE Investigators. Normalization of hemoglobin level in patients with chronic kidney disease and anemia. N Engl J Med 2006;355: 2071-2084.

[21] Mizobuchi M, Towler D, Slatopolsky E. Vascular calcification: the killer of patients with chronic kidney disease. J Am Soc Nephrol 2009;20:1453-1464.

[22] Hempel JC, Poppelaars F, Gaya da Costa M, et al. Distinct in vitro complement activation by various intravenous iron preparations. Am J Nephrol 2017;45:49-59.

[23] Zoller H, Schaefer B, Glodny B. Iron-induced hypophosphatemia: an emerging complication. Curr Opin Nephrol Hypertens 2017;26:266-275.

[24] Wolf M, Koch TA, Bregman DB. Effects of iron deficiency anemia and its treatment on fibroblast growth factor 23 and phosphate homeostasis in women. J Bone Miner Res 2013; 28:1793-1803. 